\title{
Derivation of the nonlinear fluctuating hydrodynamic equation from underdamped Langevin equation
}

\author{
Takenobu Nakamura ${ }^{1}$ and Akira Yoshimori ${ }^{2}$ \\ ${ }^{1}$ Research Institute of Computational Sciences (RICS), National Institute of \\ Advanced Industrial Science and Technology (AIST), 1-1-1 Umezono, Tsukuba \\ 305-8568, Japan \\ 2 Department of Physics, Kyushu University, Fukuoka 812-8581, Japan \\ E-mail: takenobu.nakamura@aist.go.jp
}

\begin{abstract}
We derive the fluctuating hydrodynamic equation for the number and momentum densities exactly from the underdamped Langevin equation. This derivation is an extension of the Kawasaki-Dean formula in underdamped case. The steady state probability distribution of the number and momentum densities field can be expressed by the kinetic and potential energies. In the massless limit, the obtained fluctuating hydrodynamic equation reduces to the Kawasaki-Dean equation. Moreover, the derived equation corresponds to the field equation derived from the canonical equation when the friction coefficient is zero.

PACS numbers: 05.10.Gg,05.20.Jj,05.40.-a,47.10.-g
\end{abstract}

\section{Introduction}

Field equation is widely employed in the studies on colloidal or liquid dynamics. In the study on colloidal dynamics, some researchers have applied averaged density field dynamics, which is called the time-dependent density functional method [1-7]. This method has also been successfully employed to study various phenomena observed in the field of liquid dynamics, such as solvation [7-13], transport phenomena [14], and slow relaxation in supercooled liquids $[15,16]$. Besides the average density field dynamics, other researchers have also developed theoretical expressions describing momentum density fields $[17,18]$.

As compared to the direct calculation of particle dynamics, field description is more useful for theoretical studies. This is because we can estimate many physical parameters, such as transport coefficients from the correlation functions of field variables. Thus, by using the field description, many researchers have formulated approximations for the estimation of the physical parameters. For example, the mode-coupling theory, which is known as a useful tool for approximation of the transport coefficients, has been formulated by using field description [19-22]. 
While the field description is useful for theoretical calculations, its correspondence with the particle description is not clear. Therefore, the derivation of the field description from the particle description is the fundamental problem in the studies on colloidal and liquid dynamics. When field variables are not averaged, Dean has derived the field equation from the overdamped Langevin model [23]. In a colloidal system, the timedependent density functional method can be applied for the derivation of field equations from the overdamped Langevin equation by averaging the density field on the basis of some assumptions [1-3]. Recently, a method has been developed to derive field equations from the Liouville equation describing liquid dynamics by using the projection operator method $[24,25]$. In most cases, the derivation of the field description from the particle description requires some approximations.

Very few studied have been carried out on the derivation of the field description in the nonlinear and underdamped cases. In these cases, the inertial effect has to be considered. In liquid dynamics, linear generalized Langevin equations including momentum density have been derived for the field variables of a homogeneous system [19]. Linear generalized linear Langevin equations for an inhomogeneous system have also been developed $[17,18]$. However, nonlinear equations in the filed description have not been derived. Therefore, in underdamped cases, phenomenological models have often been employed [26].

In Ref. [23], the evolution equation of the density field is derived from the overdamped Langevin equation representing the particles interacting via the pairwise potential. The derived equation is called as the 'Kawasaki-Dean formula'. In Ref. [23], a closed evolution equation for the density field is exactly derived by using Itó's formula [27], while the evolution equation is approximately derived using other field models. The steady-state probability distribution of the density field for the overdamped Langevin model is represented by the bare pairwise potential term and the entropy term. In contrast to the overdamped case, there are no exact derivations of the evolution equation for the field variables in the underdamped cases. Here, a question arises whether we can extend the Kawasaki-Dean formula to the underdamped Langevin equation. The exact derivation of the closed evolution equation for field variables is main issue of this paper.

In Sec. 2, we derive the closed evolution equation for the number density and the momentum density field using the underdamped Langevin model. For a system without dissipation, the closed evolution equation corresponds to the field equation for a Hamiltonian system. In Sec. 3, we discuss the properties of the derived evolution equation. In the Sec. 3.1, we calculate the steady state probability distribution functional of the evolution equation by using a functional Fokker-Planck equation. In Sec. 3.2, we derive the Kawasaki-Dean equation from the evolution equation derived in Sec. 2 to check the consistency between our model and other models. Sec. 4 presents the concluding remarks. 


\section{Derivation of the nonlinear fluctuating hydrodynamic equation from underdamped Langevin equation}

We study $N$ Brownian particles suspended in a three-dimensional solvent at temperature $T$. The motion of the $i$-th Brownian particle is represented by its position $\boldsymbol{x}_{i}$ and momentum $\boldsymbol{p}_{i}$, where $i=1,2, \ldots, N$ and $\boldsymbol{x}_{i} \in[0, L] \times[0, L] \times[0, L]$. We express the $\alpha$-th component of $\boldsymbol{x}_{i}$ as $x_{i}^{\alpha}$, where $\alpha=1,2$, and 3. That is, $\boldsymbol{x}_{i}=\left(x_{i}^{1}, x_{i}^{2}, x_{i}^{3}\right)$. The Brownian particles interact via the pairwise potential $V(\boldsymbol{x})$. Each Brownian particle has the same mass $m$. The motion of the $i$-th Brownian particle is described by the underdamped Langevin equation as

$$
\begin{aligned}
\frac{d \boldsymbol{x}_{i}}{d t} & =\frac{\boldsymbol{p}_{i}}{m}, \\
\frac{d \boldsymbol{p}_{i}}{d t} & =-\frac{\partial U\left(\left\{\boldsymbol{x}_{j}\right\}_{j=1}^{N}\right)}{\partial \boldsymbol{x}_{i}}-\frac{\gamma}{m} \boldsymbol{p}_{i}+\sqrt{\gamma T} \boldsymbol{R}_{i}(t),
\end{aligned}
$$

where $U\left(\left\{\boldsymbol{x}_{i}\right\}_{i=1}^{N}\right)$ denotes the total potential energy defined as

$$
U\left(\left\{\boldsymbol{x}_{i}\right\}_{i=1}^{N}\right) \equiv \frac{1}{2} \sum_{i=1}^{N} \sum_{j=1, j \neq i}^{N} V\left(\boldsymbol{x}_{i}-\boldsymbol{x}_{j}\right) .
$$

The coefficient $\gamma$ is the friction constant and $\boldsymbol{R}_{i}(t)$ is the zero-mean Gaussian white noise satisfying

$$
\left\langle R_{i}^{\alpha}(t) R_{j}^{\beta}\left(t^{\prime}\right)\right\rangle=2 \delta_{i j} \delta_{\alpha \beta} \delta\left(t-t^{\prime}\right),
$$

where $\langle\cdot\rangle$ represents the average value of $\boldsymbol{R}_{i}(t)$.

First, as described in Ref. [23], we introduce the density field $\rho(\boldsymbol{x}, t)$ given as

$$
\rho(\boldsymbol{x}, t) \equiv \sum_{i=1}^{N} \delta\left(\boldsymbol{x}-\boldsymbol{x}_{i}(t)\right) .
$$

To obtain the closed evolution equation of the fields in the underdamped system, we also introduce the momentum density fields $\boldsymbol{g}(\boldsymbol{x}, t)$ defined as

$$
\boldsymbol{g}(\boldsymbol{x}, t) \equiv \sum_{i=1}^{N} \boldsymbol{p}_{i}(t) \delta\left(\boldsymbol{x}-\boldsymbol{x}_{i}(t)\right) .
$$

One can exactly derive the closed evolution equation of the number density field and momentum density fields defined by equations (5) and (6).

Using these definitions, we derive the evolution equation using Itó's formula. Expanding the stochastic variable $\rho(\boldsymbol{x}, t)$ defined in equation $(5)$, we obtain the evolution equation for the number density as

$$
\frac{\partial \rho(\boldsymbol{x}, t)}{\partial t}=-\boldsymbol{\nabla} \cdot\left(\frac{\boldsymbol{g}(\boldsymbol{x}, t)}{m}\right)
$$

where we have used equations (1) and (6). This equation represents the continuous equation for the density field. Similarly, using equations (2) and (6), we obtain the 
evolution equation for the momentum density

$$
\begin{aligned}
\frac{\partial g^{\alpha}(\boldsymbol{x}, t)}{\partial t}= & -\frac{\gamma}{m} g^{\alpha}(\boldsymbol{x}, t)+\xi^{\alpha}(\boldsymbol{x}, t) \\
& -\rho(\boldsymbol{x}, t) \int d \boldsymbol{x} \frac{\partial V(\boldsymbol{x}-\boldsymbol{y})}{\partial x^{\alpha}} \rho(\boldsymbol{y}, t)-\frac{\partial M^{\alpha \beta}(\boldsymbol{x}, t)}{\partial x^{\beta}},
\end{aligned}
$$

where we have used Einstein's summation convention whenever a subscript is repeated in a term. Here, $\xi^{\alpha}(\boldsymbol{x}, t)$ and $M^{\alpha \beta}$ are defined as

$$
\begin{gathered}
\xi^{\alpha}(\boldsymbol{x}, t) \equiv \sum_{i=1}^{N} \sqrt{\gamma T} R_{i}^{\alpha}(t) \delta\left(\boldsymbol{x}-\boldsymbol{x}_{i}\right), \\
M^{\alpha \beta}(\boldsymbol{x}, t) \equiv \sum_{i=1}^{N} \frac{p_{i}^{\alpha}(t) p_{i}^{\beta}(t)}{m} \delta\left(\boldsymbol{x}-\boldsymbol{x}_{i}(t)\right) .
\end{gathered}
$$

Trivially, the average of $\boldsymbol{\xi}$ is zero from equation (9). Further, $\boldsymbol{\xi}$ is a multiplicative noise: time correlation depends on the instantaneous density fields. The noise term in equation (9) is rewritten in the form

$$
\xi^{\alpha}(\boldsymbol{x}, t)=\sqrt{\Gamma^{\alpha \beta}(\boldsymbol{x}, t) T} \zeta^{\beta}(\boldsymbol{x}, t),
$$

where $\Gamma^{\alpha \beta}(\boldsymbol{x}, t)$ is defined by

$$
\Gamma^{\alpha \beta}(\boldsymbol{x}, t)=\gamma \rho(\boldsymbol{x}, t) \delta_{\alpha \beta}
$$

and $\boldsymbol{\zeta}$ is the space-time Gaussian white noise satisfying

$$
\left\langle\zeta^{\alpha}(\boldsymbol{x}, t) \zeta^{\beta}\left(\boldsymbol{x}^{\prime}, t^{\prime}\right)\right\rangle=2 \delta_{\alpha \beta} \delta\left(\boldsymbol{x}-\boldsymbol{x}^{\prime}\right) \delta\left(t-t^{\prime}\right) .
$$

To obtain the closed evolution equation, we have to make the following assumption for the trajectory of the positions of a particle $\left\{\boldsymbol{x}_{i}(t)\right\}_{i=1}^{N}$ :

$$
\delta\left(\boldsymbol{x}_{i}(t)-\boldsymbol{x}_{j}(t)\right)=\delta\left(\boldsymbol{x}_{i}(t)-\boldsymbol{x}_{j}(t)\right) \delta_{i j},
$$

where $\delta_{i j}$ is the Kronecker delta. Note that our aim is to construct a map from the trajectory of the position and momentum of the particles $\left\{\boldsymbol{x}_{i}(t), \boldsymbol{p}_{i}(t)\right\}_{i=1}^{N}$ to the trajectory of density and momentum density fields $\left[\rho_{t}, \boldsymbol{g}_{t}\right] \equiv\{\rho(\boldsymbol{x}, t), \boldsymbol{g}(\boldsymbol{x}, t)\}_{\boldsymbol{x}}$. Then, equation (14) is satisfied when no two particles occupy the same position simultaneously in the mapping. Such an assumption is valid if particles interact through via a repulsive pairwise potential and a discretization of space, which is discussed in Appendix A.

Equation (10) is formally rewritten in the form

$$
\begin{aligned}
M^{\alpha \beta}(\boldsymbol{x}, t) & =\sum_{i=1}^{N} \frac{p_{i}^{\alpha}(t) p_{i}^{\beta}(t)}{m} \delta\left(\boldsymbol{x}-\boldsymbol{x}_{i}(t)\right) \frac{\sum_{j=1}^{N} \delta\left(\boldsymbol{x}-\boldsymbol{x}_{j}(t)\right)}{\sum_{k=1}^{N} \delta\left(\boldsymbol{x}-\boldsymbol{x}_{k}(t)\right)} \\
& =\frac{1}{m \rho(\boldsymbol{x}, t)} \sum_{i=1}^{N} \sum_{j=1}^{N} p_{i}^{\alpha}(t) p_{i}^{\beta}(t) \delta\left(\boldsymbol{x}-\boldsymbol{x}_{i}(t)\right) \delta\left(\boldsymbol{x}-\boldsymbol{x}_{j}(t)\right) .
\end{aligned}
$$

The infinite form included in (15) is explained in Appendix A. In the second step, we have used the definition of the density field given by the equation (5). Then, by using 
equation (14), $M^{\alpha \beta}(\boldsymbol{x}, t)$ is represented only by $\left[\rho_{t}, \boldsymbol{g}_{t}\right]$ in the form

$$
\begin{aligned}
M^{\alpha \beta}(\boldsymbol{x}, t) & =\frac{1}{m \rho(\boldsymbol{x}, t)} \sum_{i=1}^{N} p_{i}^{\alpha}(t) \delta\left(\boldsymbol{x}-\boldsymbol{x}_{i}(t)\right) \sum_{j=1}^{N} p_{j}^{\beta}(t) \delta\left(\boldsymbol{x}-\boldsymbol{x}_{j}(t)\right) \\
& =\frac{g^{\alpha}(\boldsymbol{x}, t) g^{\beta}(\boldsymbol{x}, t)}{m \rho(\boldsymbol{x}, t)}
\end{aligned}
$$

where we have used the definition of the momentum density given by equation (6) in the last step.

Finally, substituting equation (16) into equation (8), we obtain the evolution equation for the momentum density as follows:

$$
\begin{aligned}
\frac{\partial g^{\alpha}(\boldsymbol{x}, t)}{\partial t}= & -\Gamma^{\alpha \beta}(\boldsymbol{x}, t) \frac{\delta H_{K}[\rho, \boldsymbol{g}]}{\delta g^{\beta}(\boldsymbol{x}, t)}+\sqrt{\Gamma^{\alpha \beta}(\boldsymbol{x}, t) T} \zeta^{\beta}(\boldsymbol{x}, t) \\
& -\rho(\boldsymbol{x}, t) \frac{\partial}{\partial x^{\alpha}}\left(\frac{\delta H_{V}[\rho]}{\delta \rho(\boldsymbol{x}, t)}\right)-\frac{\partial}{\partial x^{\beta}}\left(\frac{g^{\alpha}(\boldsymbol{x}, t) g^{\beta}(\boldsymbol{x}, t)}{m \rho(\boldsymbol{x}, t)}\right),
\end{aligned}
$$

where we use the abbreviation for the functional derivative as

$$
\begin{aligned}
& \frac{\delta H_{V}[\rho]}{\delta \rho(\boldsymbol{x}, t)}=\left.\frac{\delta H_{V}[\varphi]}{\delta \varphi(\boldsymbol{x})}\right|_{\varphi(\boldsymbol{x})=\rho(\boldsymbol{x}, t)}, \\
& \frac{\delta H_{K}[\rho, \boldsymbol{g}]}{\delta g^{\alpha}(\boldsymbol{x}, t)}=\left.\frac{\delta H_{K}[\varphi, \boldsymbol{\psi}]}{\delta \psi^{\alpha}(\boldsymbol{x})}\right|_{\varphi(\boldsymbol{x})=\rho(\boldsymbol{x}, t), \boldsymbol{\psi}(\boldsymbol{x})=\boldsymbol{g}(\boldsymbol{x}, t)} .
\end{aligned}
$$

This abbreviation is used hereinafter. Here, $H_{V}[\varphi]$ and $H_{K}[\boldsymbol{\varphi}, \boldsymbol{\psi}]$ are functionals for the functions $\varphi(\boldsymbol{x})$ and $\boldsymbol{\psi}(\boldsymbol{x})$, respectively, and are defined as

$$
\begin{aligned}
H_{V}[\varphi] & \equiv \frac{1}{2} \int d \boldsymbol{x} \int d \boldsymbol{y} V(\boldsymbol{x}-\boldsymbol{y})(\varphi(\boldsymbol{x}) \varphi(\boldsymbol{y})-\delta(\boldsymbol{x}-\boldsymbol{y}) \varphi(\boldsymbol{x})), \\
H_{K}[\varphi, \boldsymbol{\psi}] & \equiv \int d \boldsymbol{x} \frac{\boldsymbol{\psi}(\boldsymbol{x})^{2}}{2 m \varphi(\boldsymbol{x})}
\end{aligned}
$$

Clearly, the functionals in equations (19) and (20) correspond to the internal energy and the kinetic energy of the system, respectively. Equations (7) and (17) are the desired nonlinear fluctuating hydrodynamic equations.

The first term on the right-hand side of equation (17) leads to the decay of momentum. The dissipative matrix $\Gamma^{\alpha \beta}$ in equation (17) depends on $\rho(\boldsymbol{x})$. This feature, which is a characteristic of the Brownian particle system, is in contrast to features of the Navier-Stokes equation. The dissipative matrix in the Navier-Stokes equation is given by the combination of the gradient and the shear and bulk viscosities $[21,26]$. The fluctuation-dissipation relation of the second kind is satisfied by the first and second terms on the right-hand side of equation (17). That is, the dissipative matrix is consistent with the noise coefficient.

The third and fourth terms on the right-hand side of equation (17) represent the conservative flows. The flow represented by the fourth term is caused by the momentum transfer. Further, the flow represented by the third term is caused by the gradient of the functional derivative of the Hamiltonian including the bare potential $V(\boldsymbol{x}-\boldsymbol{y}$ ) (or $\left.H_{V}[\rho]\right)$ in equation (19). This is in contrast to many field models including the chemical 
potential or free energy. The bare potential is obtained by the exact derivation from the overdamped Langevin model [23]. Thus, the present result shows that the inclusion of the bare potential is general consequence of the exact derivation without any coarse graining.

The Hamiltonian in equation (17) does not include the entropy terms, which are included in the overdamped evolution equation for the fields [23] or in the phenomenological model in the underdamped case $[7,28]$. One can drive the entropy terms for the Brownian particle system from the momentum transfer term when the overdamped limit is considered in equation (17) (Sec. 3.2). The entropy terms for liquid dynamics also originate from the momentum transfer term in the Liouville equation [25]. Those results indicates that the entropy term in the evolution equations is eliminated by explicitly treating the momentum transfer term from the point of view of the derivation from a microscopic model.

From equations (7) and (17), we also obtain the closed evolution equation of the density and momentum density from the canonical equation. Equations (1) and (2) reduced to the canonical equation when $\gamma=0$. Therefore, by substituting $\gamma=0$ into equations (7) and (17), we obtain

$$
\begin{aligned}
& \frac{\partial \rho(\boldsymbol{x}, t)}{\partial t}=-\boldsymbol{\nabla} \cdot\left[\frac{\boldsymbol{g}(\boldsymbol{x}, t)}{m}\right], \\
& \frac{\partial \boldsymbol{g}(\boldsymbol{x}, t)}{\partial t}=-\rho(\boldsymbol{x}, t) \boldsymbol{\nabla}\left[\frac{\delta H_{V}[\rho]}{\delta \rho(\boldsymbol{x}, t)}\right]-\nabla \cdot\left[\frac{\boldsymbol{g}(\boldsymbol{x}, t) \boldsymbol{g}(\boldsymbol{x}, t)}{m \rho(\boldsymbol{x}, t)}\right] .
\end{aligned}
$$

The evolution equations (21) and (22) contain the following five conserved quantities: the total energy, the total number, and total momentums. These quantities are defined as

$$
\begin{aligned}
H[\rho, \boldsymbol{g}] & \equiv H_{V}[\rho]+H_{K}[\rho, \boldsymbol{g}], \\
N[\rho] \equiv \int d \boldsymbol{x} \rho(\boldsymbol{x}), & \equiv \int \boldsymbol{x} \boldsymbol{g}(\boldsymbol{x}) .
\end{aligned}
$$

The conservation law for the total energy functional is derived as follows:

$$
\begin{aligned}
\frac{d H\left[\rho_{t}, \boldsymbol{g}_{t}\right]}{d t}= & \int d \boldsymbol{x} \frac{\partial \rho(\boldsymbol{x}, t)}{\partial t}\left[\frac{\delta H_{V}[\rho]}{\delta \rho(\boldsymbol{x}, t)}-\frac{g^{2}(\boldsymbol{x}, t)}{2 m \rho^{2}(\boldsymbol{x}, t)}\right] \\
& +\int d \boldsymbol{x} \frac{\partial g^{\alpha}(\boldsymbol{x}, t)}{\partial t} \frac{g^{\alpha}(\boldsymbol{x}, t)}{m \rho(\boldsymbol{x}, t)} .
\end{aligned}
$$

By substituting equations (21) and (22) into equation (26) and integrating by parts several times, we have

$$
\frac{d H\left[\rho_{t}, \boldsymbol{g}_{t}\right]}{d t}=-\int d \boldsymbol{x} \boldsymbol{\nabla} \cdot\left[\frac{\boldsymbol{g}(\boldsymbol{x}, t)}{m}\left(\frac{\delta H_{V}[\rho]}{\delta \rho(\boldsymbol{x}, t)}-\frac{\delta H_{K}[\rho, \boldsymbol{g}]}{\delta \rho(\boldsymbol{x}, t)}\right)\right] .
$$

The left-hand side of equation (27) is equal to zero from the divergence theorem. In addition, the conservation law for the total number can be easily checked from equation (21). 
The conservation law for the total momentum is also proved as follows:

$$
\frac{d \boldsymbol{P}\left[\boldsymbol{g}_{t}\right]}{d t}=-\int d \boldsymbol{x} \rho(\boldsymbol{x}, t) \boldsymbol{\nabla}\left[\frac{\delta H_{V}[\rho]}{\delta \rho(\boldsymbol{x}, t)}\right]-\int d \boldsymbol{x} \boldsymbol{\nabla} \cdot\left[\frac{\boldsymbol{g}(\boldsymbol{x}, t) \boldsymbol{g}(\boldsymbol{x}, t)}{m \rho(\boldsymbol{x}, t)}\right] .
$$

The first term on the right-hand side of equation (28) vanishes by the action-reaction law. The second term on the right-hand side of equation (28) vanishes from the divergence theorem. Therefore, the total momentums are conserved. Note that we have obtained the conservation law directly from the continuous model given by equations (21) and (22) without using the canonical equations (1) and (2).

Equations (21) and (22) are similar to the Euler equation in fluid mechanics [29]. In these equations, the number and the momentum are conserved, and the advection term is present. However, there are some differences between them, which will be discussed in Sec. 4.

\section{Properties of the nonlinear fluctuating hydrodynamic equation}

In this section, we discuss some aspects of the closed stochastic evolution equations (7) and (17) along with the Hamiltonians (19) and (20) and the noise given by equation (13).

\subsection{Derivation of Fokker-Planck equation for underdamped fluctuating hydrodynamic equation}

In this subsection, we calculate the steady-state probability distribution functional for the number and momentum density fields from the derived stochastic evolution equations (7) and (17). We first derive the Fokker-Planck equation for these field variables by using a standard procedure. Then, we obtain the steady-state probability distribution functional as a stationary solution for the Fokker-Planck equation. In this subsection, a time-dependent function $f(\boldsymbol{x}, t)$ is denoted by $f_{t}(\boldsymbol{x})$ using standard notations for a stochastic process.

The probability density distribution functional is defined as

$$
P([\rho, \boldsymbol{g}], t)=\left\langle\delta\left[\rho-\rho_{t}\right] \delta\left[\boldsymbol{g}-\boldsymbol{g}_{t}\right]\right\rangle,
$$

where $\langle\cdot\rangle$ represents the average over $\left\{\boldsymbol{\zeta}_{t}(\boldsymbol{x})\right\}_{\boldsymbol{x}} . \delta[\cdot]$ is a delta functional defined as

$$
\delta\left[\rho-\rho_{t}\right] \delta\left[\boldsymbol{g}-\boldsymbol{g}_{t}\right] \equiv \prod_{\boldsymbol{x}} \delta\left(\rho(\boldsymbol{x})-\rho_{t}(\boldsymbol{x})\right) \delta\left(\boldsymbol{g}(\boldsymbol{x})-\boldsymbol{g}_{t}(\boldsymbol{x})\right) .
$$

The evolution equation for the density field given by (7) is rewritten in the form

$$
d \rho_{t}(\boldsymbol{x})=-\boldsymbol{\nabla} \cdot \boldsymbol{g}_{t}(\boldsymbol{x}) d t / m \text {. }
$$

In addition, the evolution equations for the momentum density fields given by (17) is rewritten in the form

$$
d \boldsymbol{g}_{t}(\boldsymbol{x})=\boldsymbol{G}\left(\rho_{t}(\boldsymbol{x}), \boldsymbol{g}_{t}(\boldsymbol{x})\right) d t+\sqrt{\gamma \rho_{t}(\boldsymbol{x}) T} d \boldsymbol{\eta}_{t}(\boldsymbol{x}) .
$$


Here, $\boldsymbol{G}$ is defined as

$G^{\alpha}(\rho(\boldsymbol{x}), \boldsymbol{g}(\boldsymbol{x})) \equiv-\gamma \rho(\boldsymbol{x}) \frac{\delta H_{K}[\rho, \boldsymbol{g}]}{\delta g^{\alpha}(\boldsymbol{x})}-\rho(\boldsymbol{x}) \frac{\partial}{\partial x^{\alpha}}\left[\frac{\delta H_{V}[\rho]}{\delta \rho(\boldsymbol{x})}\right]-\frac{\partial}{\partial x^{\beta}}\left[\frac{g^{\alpha}(\boldsymbol{x}) g^{\beta}(\boldsymbol{x})}{m \rho(\boldsymbol{x})}\right]$

and $\eta_{t}^{\alpha}(\boldsymbol{x})$ satisfies

$$
d \eta_{t}^{\alpha}(\boldsymbol{x}) d \eta_{t}^{\beta}\left(\boldsymbol{x}^{\prime}\right)=2 \delta_{\alpha \beta} \delta\left(\boldsymbol{x}-\boldsymbol{x}^{\prime}\right) d t
$$

Here, $d \eta_{t}^{\alpha}(\boldsymbol{x}) d \eta_{t^{\prime}}^{\beta}\left(\boldsymbol{x}^{\prime}\right)$ is equal to zero in the case of $t \neq t^{\prime}$.

To obtain the Fokker-Planck equation, we apply Itó's formula in (30) as follows:

$$
\begin{aligned}
d & \left\{\delta\left[\rho-\rho_{t}\right] \delta\left[\boldsymbol{g}-\boldsymbol{g}_{t}\right]\right\} \\
= & \int d \boldsymbol{x} d \rho_{t}(\boldsymbol{x}) \frac{\delta\left\{\delta\left[\rho-\rho_{t}\right] \delta\left[\boldsymbol{g}-\boldsymbol{g}_{t}\right]\right\}}{\delta \rho_{t}(\boldsymbol{x})} \\
& +\int d \boldsymbol{x} d \boldsymbol{g}_{t}(\boldsymbol{x}) \cdot \frac{\delta\left\{\delta\left[\rho-\rho_{t}\right] \delta\left[\boldsymbol{g}-\boldsymbol{g}_{t}\right]\right\}}{\delta \boldsymbol{g}_{t}(\boldsymbol{x})} \\
& +\frac{1}{2} \int d \boldsymbol{x} d \boldsymbol{g}_{t}(\boldsymbol{x}) \cdot \frac{\delta}{\delta \boldsymbol{g}_{t}(\boldsymbol{x})}\left[\int d \boldsymbol{x}^{\prime} d \boldsymbol{g}_{t}\left(\boldsymbol{x}^{\prime}\right) \cdot \frac{\delta\left\{\delta\left[\rho-\rho_{t}\right] \delta\left[\boldsymbol{g}-\boldsymbol{g}_{t}\right]\right\}}{\delta \boldsymbol{g}_{t}\left(\boldsymbol{x}^{\prime}\right)}\right] .
\end{aligned}
$$

Here, we have defined the functional derivative as

$$
\begin{aligned}
& \int d \boldsymbol{x} d \rho_{t}(\boldsymbol{x}) \frac{\delta\left\{\delta\left[\rho-\rho_{t}\right] \delta\left[\boldsymbol{g}-\boldsymbol{g}_{t}\right]\right\}}{\delta \rho_{t}(\boldsymbol{x})} \\
& \equiv \lim _{|\Delta \boldsymbol{x}| \rightarrow 0} \sum_{\boldsymbol{I}} d \rho_{t}\left(\boldsymbol{x}_{\boldsymbol{I}}\right) \frac{\partial}{\partial \rho_{t}\left(\boldsymbol{x}_{\boldsymbol{I}}\right)} \prod_{\boldsymbol{I}^{\prime}} \delta\left(\rho_{t}\left(\boldsymbol{x}_{\boldsymbol{I}^{\prime}}\right)-\rho\left(\boldsymbol{x}_{\boldsymbol{I}^{\prime}}\right)\right) \delta\left(\boldsymbol{g}_{t}\left(\boldsymbol{x}_{\boldsymbol{I}^{\prime}}\right)-\boldsymbol{g}\left(\boldsymbol{x}_{\boldsymbol{I}^{\prime}}\right)\right),
\end{aligned}
$$

where $\boldsymbol{I}$ and $\boldsymbol{I}^{\prime}$ are the indices of the discretized space coordinate with volume $|\Delta \boldsymbol{x}|$ and $\boldsymbol{x}_{\boldsymbol{I}}$ is the discretized position. By substituting the evolution equations (31) and (32) into equation (35), we obtain

$$
\begin{aligned}
d & \left\{\delta\left[\rho-\rho_{t}\right] \delta\left[\boldsymbol{g}-\boldsymbol{g}_{t}\right]\right\} \\
= & -\int d \boldsymbol{x} \boldsymbol{\nabla} \cdot \boldsymbol{g}_{t}(\boldsymbol{x}) d t / m \frac{\delta\left\{\delta\left[\rho-\rho_{t}\right] \delta\left[\boldsymbol{g}-\boldsymbol{g}_{t}\right]\right\}}{\delta \rho_{t}(\boldsymbol{x})} \\
& +\int d \boldsymbol{x}\left(\boldsymbol{G}\left(\rho_{t}(\boldsymbol{x}), \boldsymbol{g}_{t}(\boldsymbol{x})\right) d t+\sqrt{\gamma T \rho_{t}(\boldsymbol{x})} d \boldsymbol{\eta}_{t}(\boldsymbol{x})\right) \cdot \frac{\delta\left\{\delta\left[\rho-\rho_{t}\right] \delta\left[\boldsymbol{g}-\boldsymbol{g}_{t}\right]\right\}}{\delta \boldsymbol{g}_{t}(\boldsymbol{x})} \\
& +\frac{1}{2} \int d \boldsymbol{x} \sqrt{\gamma T \rho_{t}(\boldsymbol{x})} d \boldsymbol{\eta}_{t}(\boldsymbol{x}) \\
& +\frac{\delta}{\delta \boldsymbol{g}_{t}(\boldsymbol{x})}\left[\int d \boldsymbol{x}^{\prime} \sqrt{\gamma T \rho_{t}\left(\boldsymbol{x}^{\prime}\right)} d \boldsymbol{\eta}_{t}\left(\boldsymbol{x}^{\prime}\right) \cdot \frac{\delta\left\{\delta\left[\rho-\rho_{t}\right] \delta\left[\boldsymbol{g}-\boldsymbol{g}_{t}\right]\right\}}{\delta \boldsymbol{g}_{t}\left(\boldsymbol{x}^{\prime}\right)}\right] .
\end{aligned}
$$

Changing the index of the derivative of the delta functional from $\left[\rho_{t}, \boldsymbol{g}_{t}\right]$ to $[\rho, \boldsymbol{g}]$, we obtain

$$
\begin{aligned}
& d\left\{\delta\left[\rho-\rho_{t}\right] \delta\left[\boldsymbol{g}-\boldsymbol{g}_{t}\right]\right\} \\
= & \int d \boldsymbol{x} \frac{\delta}{\delta \rho(\boldsymbol{x})}\left[\nabla \cdot \boldsymbol{g}(\boldsymbol{x}) d t / m\left\{\delta\left[\rho-\rho_{t}\right] \delta\left[\boldsymbol{g}-\boldsymbol{g}_{t}\right]\right\}\right] \\
& -\int d \boldsymbol{x} \frac{\delta}{\delta \boldsymbol{g}(\boldsymbol{x})} \cdot\left[\left(\boldsymbol{G}(\rho(\boldsymbol{x}), \boldsymbol{g}(\boldsymbol{x})) d t+\sqrt{\gamma T \rho_{t}(\boldsymbol{x})} d \boldsymbol{\eta}_{t}(\boldsymbol{x})\right)\left\{\delta\left[\rho-\rho_{t}\right] \delta\left[\boldsymbol{g}-\boldsymbol{g}_{t}\right]\right\}\right] \\
& +\int d \boldsymbol{x} \frac{\delta}{\delta \boldsymbol{g}(\boldsymbol{x})} \cdot \frac{\delta}{\delta \boldsymbol{g}(\boldsymbol{x})}\left[\gamma T \rho(\boldsymbol{x}) d t\left\{\delta\left[\rho-\rho_{t}\right] \delta\left[\boldsymbol{g}-\boldsymbol{g}_{t}\right]\right\}\right] .
\end{aligned}
$$


Here, we have integrated the third term on the right-hand side of equation (38) with respect to $\boldsymbol{x}^{\prime}$ using equation (34). By substituting equation (33) into equation (38) and averaging (38), we obtain the Fokker-Planck equation for $[\rho, \boldsymbol{g}]$ as

$$
\frac{\partial P([\rho, \boldsymbol{g}], t)}{\partial t}=\hat{\mathcal{L}}([\rho, \boldsymbol{g}]) P([\rho, \boldsymbol{g}], t) .
$$

Here, the operator $\hat{\mathcal{L}}$ is a linear operator defined as

$$
\begin{aligned}
\hat{\mathcal{L}}([\rho, \boldsymbol{g}]) \equiv & \int d \boldsymbol{x}\left\{\frac{\delta}{\delta \rho(\boldsymbol{x})} \nabla \cdot\left(\frac{\boldsymbol{g}(\boldsymbol{x})}{m}\right)\right. \\
& +\frac{\delta}{\delta \boldsymbol{g}(\boldsymbol{x})} \cdot\left[\rho(\boldsymbol{x}) \boldsymbol{\nabla}\left(\frac{\delta H_{V}[\rho]}{\delta \rho(\boldsymbol{x})}\right)+\boldsymbol{\nabla} \cdot\left(\frac{\boldsymbol{g}(\boldsymbol{x}) \boldsymbol{g}(\boldsymbol{x})}{m \rho(\boldsymbol{x})}\right)\right] \\
& \left.+\frac{\delta}{\delta \boldsymbol{g}(\boldsymbol{x})} \cdot \boldsymbol{\Gamma}(\boldsymbol{x}) \cdot\left(T \frac{\delta}{\delta \boldsymbol{g}(\boldsymbol{x})}+\frac{\delta H_{K}[\rho, \boldsymbol{g}]}{\delta \boldsymbol{g}(\boldsymbol{x})}\right)\right\},
\end{aligned}
$$

where $\Gamma^{\alpha \beta}(\boldsymbol{x})=\gamma \rho(\boldsymbol{x}) \delta_{\alpha \beta}$. We obtain the steady-state probability distribution functional $P_{\text {eq }}[\rho, \boldsymbol{g}]$ as a stationary solution of equation (39) given by

$$
P_{\mathrm{eq}}[\rho, \boldsymbol{g}]=\frac{1}{Z} \exp \left(-\frac{H_{V}[\rho]+H_{K}[\rho, \boldsymbol{g}]}{T}\right),
$$

where $Z$ is a normalization constant determined by

$$
\int \mathcal{D} \rho \mathcal{D} \boldsymbol{g} P_{\text {eq }}[\rho, \boldsymbol{g}]=1 \text {. }
$$

Note that $P_{\text {eq }}[\rho, \boldsymbol{g}]$ does not include the entropy terms although the Hamiltonian in the steady-state distribution obtained using the overdamped field model includes them [23]. Further, the steady-state distribution functional $P_{\text {eq }}[\rho, \boldsymbol{g}]$ has same form as the classical fluid [7].

\subsection{Massless limit of the underdamped fluctuating hydrodynamics}

In this subsection, from equations (7) and (17), we derive the overdamped fluctuating hydrodynamic equation for Brownian particle systems in the massless limit. In the massless limit, the obtained equation is the so-called Kawasaki-Dean formula. Note that the Kawasaki-Dean formula is derived from the overdamped Langevin equation, which is obtained from the underdamped Langevin equation in the massless limit. Therefore, the derivation of Kawasaki-Dean formula from our equations leads to a consistency between our equations and these equations. Moreover, the derivation given in this section is useful for understanding similar studies carried out in the past [7,25].

Using equations (5) and (14), we obtain the identity

$$
\rho(\boldsymbol{x}, t) \rho(\boldsymbol{x}, t)=\delta(\boldsymbol{x}-\boldsymbol{x}) \rho(\boldsymbol{x}, t) .
$$

Here, the right-hand side of the identity has an infinite value, which can be justified by the proper interpretation of discretization discussed in Appendix A. Equation (43) is satisfied only if the density is defined as the sum of delta functions. Therefore if the density is defined as a continuous function, equation (43) is not satisfied. 
By using $\tau \equiv m / \gamma$, the evolution equations of the density field and momentum density field are rewritten in the form

$$
\begin{aligned}
\frac{\partial \rho(\boldsymbol{x}, t)}{\partial t}= & -\frac{1}{\tau \gamma} \boldsymbol{\nabla} \cdot \boldsymbol{g}(\boldsymbol{x}, t) \\
\frac{\partial g^{\alpha}(\boldsymbol{x}, t)}{\partial t}= & -\frac{g^{\alpha}(\boldsymbol{x}, t)}{\tau}-\rho(\boldsymbol{x}, t) \frac{\partial}{\partial x^{\alpha}}\left[\frac{\delta H_{V}[\rho]}{\delta \rho(\boldsymbol{x}, t)}\right] \\
& -\frac{\partial}{\partial x^{\beta}}\left[\frac{g^{\alpha}(\boldsymbol{x}, t) g^{\beta}(\boldsymbol{x}, t)}{\tau \gamma \rho(\boldsymbol{x}, t)}\right]+\sqrt{\gamma T \rho(\boldsymbol{x}, t)} \zeta^{\alpha}(\boldsymbol{x}, t) .
\end{aligned}
$$

The parameter $\tau$ is the relaxation time for the density field and is constant for a given system. We focus on the time evolution of the density field whose time resolution $\Delta t$ is significantly larger than $\tau$. Then, we define

$$
\tilde{\rho}\left(\boldsymbol{x}, t_{n}\right) \equiv \lim _{\tau / \Delta t \rightarrow 0} \rho\left(\boldsymbol{x}, t_{n}\right),
$$

where $t_{n}=n \Delta t$. After taking the limit of $\tau$ and evaluating the equation, we take the continuous limit $\Delta t \rightarrow 0$ and represent the time evolution of the coarse-grained density field $\tilde{\rho}$ as follows:

$$
\begin{aligned}
\frac{\partial \tilde{\rho}(\boldsymbol{x}, t)}{\partial t} & \equiv \lim _{\Delta t \rightarrow 0}\left[\frac{\tilde{\rho}\left(\boldsymbol{x}, t_{n}+\Delta t\right)-\tilde{\rho}\left(\boldsymbol{x}, t_{n}\right)}{\Delta t}\right] \\
& =\lim _{\Delta t \rightarrow 0}\left[\lim _{\tau \rightarrow 0}\left[\frac{\rho(\boldsymbol{x}, t+\Delta t)-\rho(\boldsymbol{x}, t)}{\Delta t}\right]\right] .
\end{aligned}
$$

In the derivation of the coarse-grained evolution equation, we have used the asymptotic formula

$$
\lim _{\tau \rightarrow 0} \int_{t_{-}^{\prime}}^{t^{\prime}} d t \frac{e^{-\left(t^{\prime}-t\right) / \tau}}{\tau} A(t)=A\left(t^{\prime}\right) \text { for } t^{\prime}>t_{-}^{\prime} .
$$

That is because a term in the integrand is used in the definition of the delta function

$$
\delta\left(t-t^{\prime}\right)=\lim _{\tau \rightarrow 0} \frac{e^{-\left|t-t^{\prime}\right| / \tau}}{2 \tau} .
$$

Note that $t^{\prime}$ is the upper limit of the integral in equation (48).

By integrating (44) with respect to time, we obtain the difference $\rho(\boldsymbol{x}, t+\Delta t)-$ $\rho(\boldsymbol{x}, t)$ in equation $(47)$ as follows:

$$
\rho(\boldsymbol{x}, t+\Delta t)-\rho(\boldsymbol{x}, t)=-\frac{1}{\gamma} \nabla \cdot \int_{t}^{t+\Delta t} d t^{\prime} \frac{1}{\tau} \boldsymbol{g}\left(\boldsymbol{x}, t^{\prime}\right) .
$$

Next, we consider a system with $t \gg \tau$. By using equation (45), $\boldsymbol{g}\left(\boldsymbol{x}, t^{\prime}\right)$ is formally solved as

$$
\frac{1}{\tau} \boldsymbol{g}\left(\boldsymbol{x}, t^{\prime}\right)=\frac{1}{\tau} \boldsymbol{g}(\boldsymbol{x}, 0) e^{-t^{\prime} / \tau}+\boldsymbol{\Upsilon}\left(\boldsymbol{x}, t^{\prime}\right)+\boldsymbol{\Xi}\left(\boldsymbol{x}, t^{\prime}\right)+\boldsymbol{\Pi}\left(\boldsymbol{x}, t^{\prime}\right) .
$$

Here, we have introduced the quantities

$$
\Upsilon^{\alpha}\left(\boldsymbol{x}, t^{\prime}\right) \equiv-\frac{1}{\tau} \int_{0}^{t^{\prime}} d s e^{-\left(t^{\prime}-s\right) / \tau} \rho(\boldsymbol{x}, s) \frac{\partial}{\partial x^{\alpha}}\left[\frac{\delta H_{V}[\rho]}{\delta \rho(\boldsymbol{x}, s)}\right],
$$




$$
\begin{aligned}
& \Xi^{\alpha}\left(\boldsymbol{x}, t^{\prime}\right) \equiv \frac{1}{\tau} \int_{s=0}^{s=t^{\prime}} e^{-\left(t^{\prime}-s\right) / \tau} \sqrt{\gamma T \rho(\boldsymbol{x}, s)} d \eta_{s}^{\alpha}(\boldsymbol{x}), \\
& \Pi^{\alpha}\left(\boldsymbol{x}, t^{\prime}\right) \equiv-\frac{1}{\tau} \int_{0}^{t^{\prime}} d s e^{-\left(t^{\prime}-s\right) / \tau} \frac{\partial}{\partial x^{\beta}}\left[\frac{g^{\alpha}(\boldsymbol{x}, s) g^{\beta}(\boldsymbol{x}, s)}{\tau \gamma \rho(\boldsymbol{x}, s)}\right] .
\end{aligned}
$$

The terms $\boldsymbol{\Xi}, \boldsymbol{\Upsilon}$, and $\boldsymbol{\Pi}$ correspond to noise, drift, and advection terms respectively. Substituting equation (51) into equation (50), we obtain

$\gamma(\rho(\boldsymbol{x}, t+\Delta t)-\rho(\boldsymbol{x}, t))=-\boldsymbol{\nabla} \cdot\left[\int_{t}^{t+\Delta t} d t^{\prime}\left(\boldsymbol{\Upsilon}\left(\boldsymbol{x}, t^{\prime}\right)+\boldsymbol{\Xi}\left(\boldsymbol{x}, t^{\prime}\right)+\boldsymbol{\Pi}\left(\boldsymbol{x}, t^{\prime}\right)\right)\right]$.

Using equations (52), (53), (54), and (55), we evaluate the right-hand side of equation (47) as follows. First, we substitute equations (52), (53), and (54) recursively into the left-hand side of equation (55). Next, taking the limit $\tau \rightarrow 0$, we evaluate it to the first order of $\Delta t$. Then, taking the continuous limit $\Delta t \rightarrow 0$, we obtain the right-hand side of equation (47).

First, we integrate of $\Upsilon$. By using equation (48), equation (52) is evaluated as

$$
\lim _{\tau \rightarrow 0} \Upsilon^{\alpha}\left(\boldsymbol{x}, t^{\prime}\right)=-\tilde{\rho}\left(\boldsymbol{x}, t^{\prime}\right) \frac{\partial}{\partial x^{\alpha}}\left[\frac{\delta H_{V}[\tilde{\rho}]}{\delta \tilde{\rho}\left(\boldsymbol{x}, t^{\prime}\right)}\right] .
$$

Then, the integral of $\Upsilon\left(\boldsymbol{x}, t^{\prime}\right)$ in the limit $\tau \rightarrow 0$ is evaluated as

$$
\lim _{\tau \rightarrow 0} \int_{t}^{t+\Delta t} d t^{\prime} \Upsilon^{\alpha}\left(\boldsymbol{x}, t^{\prime}\right)=-\tilde{\rho}(\boldsymbol{x}, t) \frac{\partial}{\partial x^{\alpha}}\left[\frac{\delta H_{V}[\tilde{\rho}]}{\delta \tilde{\rho}(\boldsymbol{x}, t)}\right] \Delta t .
$$

Next, to integrate $\boldsymbol{\Xi}$, we calculate the correlation for these variables in the case of $\Delta t \gg \tau$. The product $\Xi^{\alpha}\left(\boldsymbol{x}, t_{1}\right) \Xi^{\beta}\left(\boldsymbol{x}^{\prime}, t_{2}\right)$ is calculated as

$$
\begin{aligned}
\Xi^{\alpha}\left(x, t_{1}\right) \Xi^{\beta}\left(x^{\prime}, t_{2}\right) & =\int_{s_{1}=0}^{s_{1}=t_{1}} \int_{s_{2}=0}^{s_{2}=t_{2}} \frac{e^{-\left(t_{1}+t_{2}-s_{1}-s_{2}\right) / \tau}}{\tau^{2}} \gamma T \sqrt{\rho\left(\boldsymbol{x}, s_{1}\right) \rho\left(\boldsymbol{x}^{\prime}, s_{2}\right)} d \eta_{s_{1}}^{\alpha}(\boldsymbol{x}) d \eta_{s_{2}}^{\beta}\left(\boldsymbol{x}^{\prime}\right) \\
& =2 \gamma T \delta\left(\boldsymbol{x}-\boldsymbol{x}^{\prime}\right) \delta^{\alpha \beta} e^{-\left|t_{1}-t_{2}\right| / \tau} \int_{0}^{\min \left[t_{1}, t_{2}\right]} d s \frac{e^{-2\left(\min \left[t_{1}, t_{2}\right]-s\right) / \tau}}{\tau^{2}} \rho(\boldsymbol{x}, s) .(58)
\end{aligned}
$$

Here, we have used Itó calculus (34) and the identity $t_{1}+t_{2}=\left|t_{1}-t_{2}\right|+2 \min \left[t_{1}, t_{2}\right]$. By integrating equation (58) with respect to time $t_{1}$ and $t_{2}$, we represent the product of integrations of $\Xi$ as

$$
\begin{aligned}
\int_{t}^{t+\Delta t} d t_{1} \int_{t}^{t+\Delta t} d t_{2} \Xi^{\alpha}\left(\boldsymbol{x}, t_{1}\right) \Xi^{\beta}\left(\boldsymbol{x}^{\prime}, t_{2}\right)= & 2 \gamma T \delta_{\alpha \beta} \delta\left(\boldsymbol{x}-\boldsymbol{x}^{\prime}\right) \int_{t}^{t+\Delta t} d t_{1} \int_{t}^{t+\Delta t} d t_{2} \frac{e^{-\left|t_{1}-t_{2}\right| / \tau}}{\tau} \\
& \times \int_{0}^{\min \left[t_{1}, t_{2}\right]} d s \frac{e^{-2\left(\min \left[t_{1}, t_{2}\right]-s\right) / \tau}}{\tau} \rho(\boldsymbol{x}, s) .
\end{aligned}
$$

Taking the limit $\tau \rightarrow 0$ in equation (59), we obtain

$\lim _{\tau \rightarrow 0} \int_{t}^{t+\Delta t} d t_{1} \int_{t}^{t+\Delta t} d t_{2} \Xi^{\alpha}\left(\boldsymbol{x}, t_{1}\right) \Xi^{\beta}\left(\boldsymbol{x}^{\prime}, t_{2}\right)=2 \gamma T \delta_{\alpha \beta} \delta\left(\boldsymbol{x}-\boldsymbol{x}^{\prime}\right) \tilde{\rho}(\boldsymbol{x}, t) \Delta t+o(\Delta t)$.

Comparing equation (60) with equations (11), (12), and (13), we find that the time average of $\boldsymbol{\Xi}(\boldsymbol{x}, t)$ coincides with that of $\boldsymbol{\xi}(\boldsymbol{x}, t)$ when $\rho$ is replaced with $\tilde{\rho}$. 
Next, we integrate of $\Pi$. By substituting equation (51) into equation (54) recursively, we integrate of $\Pi\left(\boldsymbol{x}, t^{\prime}\right)$ as follows:

$$
\begin{aligned}
\int_{t}^{t+\Delta t} d t^{\prime} \Pi^{\alpha}\left(\boldsymbol{x}, t^{\prime}\right) & =-\frac{\partial}{\partial x^{\beta}}\left[\int_{t}^{t+\Delta t} d t^{\prime} \int_{0}^{t^{\prime}} d s e^{-\left(t^{\prime}-s\right) / \tau} \frac{1}{\gamma \rho(\boldsymbol{x}, s)}\left(\frac{g^{\alpha}(\boldsymbol{x}, s)}{\tau}\right)\left(\frac{g^{\beta}(\boldsymbol{x}, s)}{\tau}\right)\right] \\
& =-\frac{\partial}{\partial x^{\beta}}\left[\int_{t}^{t+\Delta t} d t^{\prime} \int_{0}^{t^{\prime}} d s e^{-\left(t^{\prime}-s\right) / \tau} \frac{\Xi^{\alpha}(\boldsymbol{x}, s) \Xi^{\beta}(\boldsymbol{x}, s)}{\gamma \rho(\boldsymbol{x}, s)}\right]+o(\Delta t) . \quad(61)
\end{aligned}
$$

In the second step, we have used the estimation $\Xi^{\alpha}(\boldsymbol{x}, t) \propto \tau^{-1 / 2}$ obtained from the following identity:

$$
\Xi^{\alpha}\left(\boldsymbol{x}, t_{1}\right) \Xi^{\beta}\left(\boldsymbol{x}, t_{1}\right)=2 \gamma T \delta_{\alpha \beta} \int_{0}^{t_{1}} d s \frac{e^{-2\left(t_{1}-s\right) / \tau}}{\tau^{2}}[\rho(\boldsymbol{x}, s)]^{2},
$$

which is obtained by substituting $t_{1}=t_{2}$ and $\boldsymbol{x}=\boldsymbol{x}^{\prime}$ into equation (58) using equation (43).

Substituting equation (62) into equation (61), the integration of $\Pi$ is given as follows:

$$
\begin{aligned}
& \int_{t}^{t+\Delta t} d t^{\prime} \Pi^{\alpha}\left(\boldsymbol{x}, t^{\prime}\right) \\
& =-\frac{\partial}{\partial x^{\alpha}}\left[\int_{t}^{t+\Delta t} d t^{\prime} \int_{0}^{t^{\prime}} d s e^{-\left(t^{\prime}-s\right) / \tau} \frac{1}{\gamma \rho(\boldsymbol{x}, s)} \int_{0}^{s} d s_{1} \frac{e^{-2\left(s-s_{1}\right) / \tau}}{\tau^{2}} 2 \gamma T\left[\rho\left(\boldsymbol{x}, s_{1}\right)\right]^{2}\right] \\
& +o(\Delta t) .
\end{aligned}
$$

Taking the limit $\tau \rightarrow 0$ in equation (63) and by using the identity (48), we obtain

$$
\lim _{\tau \rightarrow 0} \int_{t}^{t+\Delta t} d t^{\prime} \boldsymbol{\Pi}\left(\boldsymbol{x}, t^{\prime}\right)=-T \nabla \tilde{\rho}(\boldsymbol{x}, t) \Delta t+o(\Delta t) .
$$

This evaluation shows that the coarse graining of the advection term yields the diffusion term in the fluctuating hydrodynamics model of Brownian dynamics.

Finally, we obtain the change in density from time $t$ to $t+\Delta t$ in the limit of $\tau \rightarrow 0$ by substituting equations (57) and (64) into equation (55). The change in density to the order of $\Delta t$ is written as

$$
\begin{aligned}
\lim _{\tau \rightarrow 0}[\gamma(\rho(\boldsymbol{x}, t+\Delta t)-\rho(\boldsymbol{x}, t))]= & -\Delta t \boldsymbol{\nabla} \cdot\left[\tilde{\rho}(\boldsymbol{x}, t) \boldsymbol{\nabla}\left[-\frac{\delta H[\tilde{\rho}]}{\delta \tilde{\rho}(\boldsymbol{x}, t)}\right]-T \boldsymbol{\nabla} \tilde{\rho}(\boldsymbol{x}, t)\right] \\
& -\lim _{\tau \rightarrow 0} \boldsymbol{\nabla} \cdot \int_{t}^{t+\Delta t} d t^{\prime} \boldsymbol{\Xi}\left(\boldsymbol{x}, t^{\prime}\right)+o(\Delta t) .
\end{aligned}
$$

By multiplying both sides of equation (65) by $\Delta t^{-1}$ and taking the limit $\Delta t \rightarrow 0$, we obtain

$\frac{\partial \tilde{\rho}(\boldsymbol{x}, t)}{\partial t}=-\frac{1}{\gamma} \boldsymbol{\nabla} \cdot\left[-\tilde{\rho}(\boldsymbol{x}, t) \nabla\left[\frac{\delta H_{V}[\tilde{\rho}]}{\delta \tilde{\rho}(\boldsymbol{x}, t)}\right]-T \nabla \tilde{\rho}(\boldsymbol{x}, t)+\sqrt{\gamma T \tilde{\rho}(\boldsymbol{x}, t)} \boldsymbol{\zeta}(\boldsymbol{x}, t)\right]$.

Here, we can rewrite the noise term as

$$
\lim _{\Delta t \rightarrow 0}\left[\lim _{\tau \rightarrow 0} \frac{1}{\Delta t} \int_{t}^{t+\Delta t} d t^{\prime} \boldsymbol{\Xi}\left(\boldsymbol{x}, t^{\prime}\right)\right]=\sqrt{\gamma T \tilde{\rho}(\boldsymbol{x}, t)} \boldsymbol{\zeta}(\boldsymbol{x}, t)
$$


because (60) shows that intensity in the left hand side of (67) coincides with that in the right hand side of (67). This is the fluctuating hydrodynamic equation for the density in the overdamped limit [23]. Using a technique similar to that used in the underdamped case, we can obtain the steady-state distribution function written as

$$
P_{\text {eq }}[\tilde{\rho}] \propto \exp \left(-\frac{H_{V}[\tilde{\rho}]}{T}-\int d \boldsymbol{x} \tilde{\rho}(\boldsymbol{x})(\log \tilde{\rho}(\boldsymbol{x})-1)\right) .
$$

From the derivation of the Kawasaki-Dean formula (66), we have found that diffusion is caused by the advection due to a random force. In contrast, in the case of liquids, diffusion is caused by the liquid itself. The physical origin of the diffusion term obtained by our model, therefore, is different from that in a liquid system although the expressions of in both the cases appear similar.

\section{Discussion}

The primary objective of this study is the derivation of the underdamped nonlinear fluctuating hydrodynamic equation (17) along with equations (7), (13), (19), and (20). The starting point is the underdamped Langevin equations (1) and (2). It is a nontrivial fact that we obtain the closed stochastic evolution equation of the density field and momentum density fields from the particle description model. The exact derivation would have been obtained if we had taken the continuous limit using the discretization scheme discussed in Appendix A. The obtained evolution equation is reasonable because the Fokker-Planck equation obtained using our model agrees with that obtained using a classical liquid system, except for the form of the dissipative matrix [7].

The underdamped equation is unrealistic when describing the experimental situation of Brownian particle system. The overdamped model is more suitable as compared to the underdamped model, for a Brownian particle system. Nevertheless, the underdamped model is useful for theoretical approximations such as the modecoupling theory. The underdamped model for the Brownian particles can be a basis for the derivation of the mode coupling equation [30]. Recently, some researchers have developed systematic methods for the derivation of the mode-coupling equation from the overdamped model for a Brownian particle system [31]. However, the derived equation is slightly different from the mode-coupling equation [31]. The difference might be eliminated if the mode-coupling equation is derived from the underdamped model.

In addition, the underdamped model fits a liquid system. The moment of liquid particles should be explicitly treated in order to study the phenomena observed before momentum relaxation. In a liquid system, however, the field description in the nonlinear and underdamped cases has not been intensively studied. The present equations (21) and (22) for $\gamma=0$ can be applied in this case. They are useful for microscopic studies of a liquid system.

There are similarities and differences between our equations and the Euler equation. A point $\boldsymbol{x}$ in equations (21) and (22) includes not more than one particle. In contrast, a point $\boldsymbol{x}$ in the Euler equation includes many particles such that the 
thermodynamic variables are well defined. Equations (21) and (22) have been derived exactly, except for the condition (14). In addition, Euler equation is based on the local equilibrium assumption. In contrast, equations (21) and (22) can be derived without such assumptions. Thus, equations (21) and (22) can be used to describe the liquid that is not in the local equilibrium state.

In Sec. 3.2, we have derived the Kawasaki-Dean formula by coarse-graining our model with respect to time under the condition (43). Similar coarse graining methods for the Fokker-Planck equation for the derivation of the equation of the density and momentum density describing a liquid system have been devised [7]. The coarsegraining method described in Ref. [7] does not require the condition (43). Therefore, the condition (43) is not required if we carry our coarse graining for the Fokker-Planck equation derived in Sec. 3.1. This will be investigated in our future study.

We have found inconsistencies between the steady-state probability distributions (41) and (68). In Sec. 3.1, we have derived the steady-state probability distribution (41) for the underdamped model. We have also obtained the steady-state probability distribution for the overdamped model by using equation (68). The probability distribution (68) is not obtained by integrating equation (41) with respect to $[\boldsymbol{g}]$. We guess that the inconsistencies might be related to the singularity of the delta function in equations (5) and (6). However, the relation between the inconsistencies and the singularity has not determined thus far. We will address these inconsistencies in our future study.

\section{Acknowledgments}

We are grateful to H. Furusawa, K. Miyazaki, S. Sasa, and Y. Hyuga for their helpful discussions of this work. This study was supported by the Grant-in-Aid for Scientific Research on Priority Area and for Scientific Research (C) from the Japanese Ministry of Education, Science, Sports and Culture.

\section{Appendix A. Justification of equations (14), (15) (16) and (43)}

\section{Appendix A.1. Discretization method and justification of (14)}

In this study, we have often treated the delta function in the manner which is not mathematically well defined. In this section, we give the correct interpretation of these treatments and representations.

First, we design a discretized cell $|\Delta \boldsymbol{x}|$ that has a finite size is so small that different particles cannot occupy the same cell. Such a situation can be considered if the potential has a repulsive core within a short length $r_{c}$. We denote the position of the cell by $\boldsymbol{I}$ introduced in Sec. 3.1. Because the cell size is sufficiently small, the map from $i$ to $\boldsymbol{I}$ is an injective map. Then, the following equality is satisfied:

$$
\frac{1}{|\Delta \boldsymbol{x}|} \delta_{\left\lfloor\boldsymbol{x}_{i} / \Delta \boldsymbol{x}\right\rfloor,\left\lfloor\boldsymbol{x}_{j} / \Delta \boldsymbol{x}\right\rfloor}=\frac{1}{|\Delta \boldsymbol{x}|} \delta_{\left\lfloor\boldsymbol{x}_{i} / \Delta \boldsymbol{x}\right\rfloor,\left\lfloor\boldsymbol{x}_{j} / \Delta \boldsymbol{x}\right\rfloor} \delta_{i, j},
$$


where $\lfloor a\rfloor$ is Gauss's notation representing the maximum integer less than $a$ and $\delta_{\left\lfloor\boldsymbol{x}_{i} / \Delta \boldsymbol{x}\right\rfloor,\left\lfloor\boldsymbol{x}_{j}(t) / \Delta \boldsymbol{x}\right\rfloor} \equiv \prod_{\alpha=1,2,3} \delta_{\left\lfloor x_{i, \alpha}(t) / \Delta x^{\alpha}\right\rfloor,\left\lfloor x_{j, \alpha}(t) / \Delta x^{\alpha}\right\rfloor}$. When we take the continuous limit $|\Delta x| / r_{c}^{3} \rightarrow 0$, equation (A.1) converges to equation (14).

\section{Appendix A.2. Justification of equations (15) and (16)}

The evaluation of equation (15) leads to its infinite form. This infinite form is also justified by discretization. First, we represent $\rho$ in equation (5), $g^{\alpha}$ in equation (6) and $M^{\alpha \beta}$ in equation (10) in the discretized form as follows:

$$
\begin{aligned}
\rho_{\boldsymbol{I}, t} & =\sum_{i=1}^{N} \frac{\delta_{\boldsymbol{I},\left\lfloor\boldsymbol{x}_{i}(t) / \Delta \boldsymbol{x}\right\rfloor}}{|\Delta \boldsymbol{x}|}, \\
g_{\boldsymbol{I}, t}^{\alpha} & =\sum_{i=1}^{N} p_{i}^{\alpha}(t) \frac{\delta_{\boldsymbol{I},\left\lfloor\boldsymbol{x}_{i}(t) / \Delta \boldsymbol{x}\right\rfloor}}{|\Delta \boldsymbol{x}|}, \\
M_{\boldsymbol{I}, t}^{\alpha \beta} & =\sum_{i=1}^{N} \frac{p_{i}^{\alpha}(t) p_{i}^{\beta}(t)}{m} \frac{\delta_{\boldsymbol{I},\left\lfloor\boldsymbol{x}_{i}(t) / \Delta \boldsymbol{x}\right\rfloor}}{|\Delta \boldsymbol{x}|} .
\end{aligned}
$$

Trivially, by taking the continuous limit mentioned above, $\rho_{\boldsymbol{I}, t}$ and $\boldsymbol{g}_{\boldsymbol{I}, t}$ converges to $\rho(\boldsymbol{x}, t)$ and $\boldsymbol{g}(\boldsymbol{x}, t)$, respectively.

Using these discretized forms and by dividing the cell position $\boldsymbol{I}$ into two cases, we will prove that

$$
M_{\boldsymbol{I}, t}^{\alpha \beta}=\frac{g_{\boldsymbol{I}, t}^{\alpha} g_{\boldsymbol{I}, t}^{\beta}}{m \rho_{\boldsymbol{I}, t}} .
$$

In the first case, consider $i$ such that $\boldsymbol{I}=\left\lfloor\boldsymbol{x}_{i}(t) / \Delta \boldsymbol{x}\right\rfloor$. In the second case, $\boldsymbol{I} \neq$ $\left\lfloor\boldsymbol{x}_{i}(t) / \Delta \boldsymbol{x}\right\rfloor$ at any value of $i$. Equation (A.5) corresponds to equation (16) in the continuous limit.

In the case of $\boldsymbol{I}=\left\lfloor\boldsymbol{x}_{i}(t) / \Delta \boldsymbol{x}\right\rfloor$, we can prove that the left- and right-hand sides of equation (A.5) are equivalent. In this case, we can calculate the left-hand side of equation (A.5) from equation (A.4), so that

$$
M_{\boldsymbol{I}, t}^{\alpha \beta}=\frac{p_{i}^{\alpha}(t) p_{i}^{\beta}(t)}{m|\Delta \boldsymbol{x}|} .
$$

Since equations (A.2) and (A.3) reduce to $\rho_{\boldsymbol{I}, t}=1 /|\Delta \boldsymbol{x}|$ and $g_{\boldsymbol{I}, t}^{\alpha}=p_{i}^{\alpha}(t) /|\Delta \boldsymbol{x}|$ respectively, we have

$$
\frac{g_{\boldsymbol{I}, t}^{\alpha} g_{\boldsymbol{I}, t}^{\beta}}{m \rho_{\boldsymbol{I}, t}}=\left(\frac{p_{i}^{\alpha}(t)}{|\Delta \boldsymbol{x}|}\right)\left(\frac{p_{i}^{\beta}(t)}{|\Delta \boldsymbol{x}|}\right)\left(\frac{|\Delta \boldsymbol{x}|}{m}\right)=\frac{p_{i}^{\alpha}(t) p_{i}^{\beta}(t)}{m|\Delta \boldsymbol{x}|} .
$$

Equations (A.6) and (A.7) are equivalent.

In the case of $\boldsymbol{I} \neq\left\lfloor\boldsymbol{x}_{i}(t) / \Delta \boldsymbol{x}\right\rfloor$ at any value of $i$, we prove equation (A.5) by defining the right-hand side by zero. From equation (A.4) and $\delta_{I^{\alpha},\left\lfloor x_{i}^{\alpha}(t) / \Delta x\right\rfloor}=0$, we obtain $M_{\boldsymbol{I}, t}^{\alpha \beta}=0$. The right-hand side of equation (A.5) is defined by zero although it has an infinite form because $\rho_{\boldsymbol{I}, t}=0$. 
Appendix A.3. Justification of equation (43)

The left-hand side of equation (43) is not well defined mathematically because of the singularity. This singularity is eliminated by the discretization of equation (43). By using equation (A.2), the product of $\rho_{\boldsymbol{I}, t}$ is easily calculated as

$$
\rho_{\boldsymbol{I}, t} \rho_{\boldsymbol{I}, t}=\frac{1}{|\Delta \boldsymbol{x}|^{2}} \sum_{i, j=1}^{N} \delta_{\boldsymbol{I},\left\lfloor\boldsymbol{x}_{i}(t) / \Delta \boldsymbol{x}\right\rfloor} \delta_{\left\lfloor\boldsymbol{x}_{i}(t) / \Delta \boldsymbol{x}\right\rfloor,\left\lfloor\boldsymbol{x}_{j}(t) / \Delta \boldsymbol{x}\right\rfloor} .
$$

Substituting equation (A.1) into equation (A.8) and taking the summation with respect to $j$, we obtain

$$
\rho_{\boldsymbol{I}, t} \rho_{\boldsymbol{I}, t}=\frac{1}{|\Delta \boldsymbol{x}|^{2}} \sum_{i=1}^{N} \delta_{\boldsymbol{I},\left\lfloor\boldsymbol{x}_{i}(t) / \Delta \boldsymbol{x}\right\rfloor}
$$

In the continuous limit, equation (A.9) corresponds to equation (43).

\section{Reference}

[1] Marconi U M B and Tarazona P 1999 J. Chem. Phys. 1108032

[2] Archer A J and Evans R 2004 J. Chem. Phys. 1214246

[3] Marconi U M B and Tarazona P 2000 J. Phys.: Condens. Matter 12 A413

[4] Penna F and Tarazona P 2003 J. Chem. Phys. 1191766

[5] Penna F, Dzubiella J and Tarazona P 2003 Phys. Rev. E 68061407

[6] Dzubiella J and Likos C N 2003 J. Phys.: Condens. Matter 15 L147

[7] Kawasaki K 1994 Physica A 20835

[8] Bagchi B and Chandra A 1988 Proc. Indian Acad. Sci. (Chem. Sci.) 100353

[9] Chandra A and Bagchi B 1988 Chem. Phys. Letters 15147

[10] Yoshimori A 1996 J. Chem. Phys. 1055971

[11] Yoshimori A, Day T J F and Patey G N 1998 J. Chem. Phys. 1086378

[12] Yoshimori A, Day T J F and Patey G N 1998 J. Chem. Phys. 1093222

[13] Yoshimori A 2004 Journal of Theoretical and Computational Chemistry 3117

[14] Araki J and Munakata T 1995 Phys. Rev. E 522577

[15] Fuchizaki K and Kawasaki K 1998 J. Phys. Soc. Jpn. 671505

[16] Fuchizaki K and Kawasaki K 1998 J. Phys. Soc. Jpn. 672158

[17] Yamaguchi T, Matsuoka T and Koda S 2005 J. Chem. Phys. 123034504

[18] Yamaguchi T, Matsuoka T and Koda S 2007 J. Mol. Liq. 1341

[19] Hansen J P and MacDonald I R 1986 Theory of Simple Liquids (London: Academic Press)

[20] Sjögren L and Sjölander A 1979 J. Phys. C 124369

[21] Kirkpatrick T R and Nieuwoudt J C 1986 Phys. Rev. A 332651

[22] Kirkpatrick T R and Nieuwoudt J C 1986 Phys. Rev. A 332658

[23] Dean D S 1996 J Phys. A: Math. Gen 29 L613.

[24] Yoshimori A 1999 Phys. Rev. E 596535

[25] Yoshimori A 2005 Phys. Rev. E 71031203

[26] Das S P and Mazenko G F 1986 Phys. Rev. A 342265

[27] Gardiner C W 1990 Handbook of Stochastic Methods for Physics, Chemistry, and the Natural Sciences (Berlin: Springer)

[28] Taka H. Nishino and Hisao Hayakawa http://jp.arxiv.org/abs/0803.1797v1

[29] Landau L. D and Lifshitz E M 1987 Fluid Mechanics (Oxford: Butterworth-Heinemann)

[30] Zaccarelli E, Foffi G, Sciortino F, Tartaglia P and Dawson K A 2001 Europhys. Lett. 55(2) 157

[31] Kim B and Kawasaki K 2007 J. Phys. A: Math. Gen. 40 F33 